Bull. Austral. Math. Soc.

$31 \mathrm{C} 15,28 \mathrm{~A} 12,54 \mathrm{D} 45$

VOL. 72 (2005) [423-440]

\title{
RENDEZVOUS NUMBERS IN NORMED SPACES
}

\author{
Bálint Farkas and SZILÁRd György RÉvÉsZ
}

\begin{abstract}
In previous papers, we used abstract potential theory, as developed by Fuglede and Ohtsuka, to a systematic treatment of rendezvous numbers. We considered Chebyshev constants and energies as two variable set functions, and introduced a modified notion of rendezvous intervals which proved to be rather nicely behaved even for only lower semicontinuous kernels or for not necessarily compact metric spaces.

Here we study the rendezvous and average numbers of possibly infinite dimensional normed spaces. It turns out that very general existence and uniqueness results hold for the modified rendezvous numbers in all Banach spaces. We also observe the connections of these magical numbers to Chebyshev constants, Chebyshev radius and entropy. Applying the developed notions with the available methods we calculate the rendezvous numbers or rendezvous intervals of certain concrete Banach spaces. In particular, a satisfactory description of the case of $L_{p}$ spaces is obtained for all $p>0$.
\end{abstract}

\section{INTRODUCTION}

It was shown by Gross [13] that for a compact, connected metric space $(X, d)$ there exists a unique number $r:=r(X) \in \mathbb{R}$ such that for each finite point system $P=\left\{x_{1}, x_{2}, \ldots, x_{n}\right\} \subset X, n \in \mathbb{N}$ one finds a point $x \in X$ with the average distance to $P$ being exactly $r$, that is

$$
\frac{1}{n} \sum_{i=1}^{n} d\left(x, x_{i}\right)=r
$$

This number $r(X)$ is called the rendezvous number of the space $X$. Using this strict definition, that is requiring the very existence of a point $x$ with exact equality under (1), it is well-known that both compactness and connectedness are crucial assumptions. However, one can relax the requirements by considering so-called weak rendezvous numbers, meaning that there exist two points $y, z \in X$ with their average distances to the points $x_{j}$ being less or equal than $r$ and greater or equal than $r$, respectively, [22]. Clearly,

Received 11th July, 2005

The present publication was supported by the Hungarian-French Scientific and Technological Governmental Cooperation, project \# F-10/04 and the Hungarian-Spanish Scientific and Technological Governmental Cooperation, project \# E-38/04.

Copyright Clearance Centre, Inc. Serial-fee code: 0004-9727/05 \$A2.00+0.00. 
for connected spaces this is equivalent to the existence of a strong rendezvous number. Hence it is not surprising that one can prove the existence and uniqueness of such weak rendezvous numbers under the hypothesis of compactness, see for example, [22]. However, dropping the compactness condition one can not expect uniqueness as, for example, the case of $C(K)$ spaces shows $([11,16])$. Furthermore, sticking to connected spaces but relaxing compactness we cannot prove the existence of rendezvous numbers. For example, the unit spheres of $\ell_{p}$ spaces have no rendezvous number (unless $p=2,+\infty$ ) $([14,15,16,24])$.

In [6] we employed a systematic potential theoretic approach to rendezvous numbers and introduced a modified definition of these numbers, considering also closure of the occurring average distance sets in the construction. In the classical case of compact sets and continuous kernels (for example, distances on compact spaces), closure is superfluous as a continuous image of a compact set is also compact, hence closed. However, in the more general case of non-compact sets, like unit spheres of infinite dimensional Banach spaces, and also in case of more general, only lower semicontinuous kernels, this approach is productive. In particular, with the new definition we have found very general existence results, far beyond the setting of metric spaces. It turned out that abstract potential theory on locally compact spaces with a lower semicontinuous kernel is an appropriate framework for such investigations. In [6] it was also indicated that the local compactness assumption on the space $X$ is not necessary and the results go through to metric, but not necessarily locally compact spaces as well.

We analysed further consequences of this approach in the context of metric spaces in [7], extending and explaining a good deal of the previous knowledge. In the present paper we continue the study of rendezvous - and average numbers in normed spaces.

Let us fix some notation and introduce the necessary concepts. In the abstract potential theory developed by Fuglede [9] and Ohtsuka [17] the usual assumptions are the following. $X$ is a locally compact, Hausdorff space and $k: X \times X \rightarrow \mathbb{R}_{+} \cup\{+\infty\}$ is a lower semicontinuous, symmetric, positive kernel function. Nevertheless, we shall consider possibly infinite dimensional Banach spaces, so the local compactness assumption needs to be relaxed. We shall accomplish this task on the cost of allowing special kernel functions only, such as $k(x, y):=\|x-y\|$, which is just the usual kernel appearing in connection with rendezvous numbers.

Definition 1: For arbitrary $H, L \subset X$ the general $n^{\text {th }}$ Chebyshev constant of $L$ with respect to $H$ is defined as

$$
M_{n}(H, L):=\sup _{w_{1}, \ldots, w_{n} \in H} \inf _{x \in L} \frac{1}{n}\left(\sum_{k=1}^{n} k\left(x, w_{k}\right)\right),
$$


and the $n^{\text {th }}$ general dual Chebyshev constant of $L$ relative to $H$ is

$$
\bar{M}_{n}(H, L):=\inf _{w_{1}, \ldots, w_{n} \in H} \sup _{x \in L} \frac{1}{n}\left(\sum_{j=1}^{n} k\left(x, w_{j}\right)\right) .
$$

The first part of the definition is due to Ohtsuka [18]. By standard considerations, just as in the case of classical Chebyshev constants, one sees that $M_{n}(H, L)$ and $\bar{M}_{n}(H, L)$ converge to some $M(H, L), \bar{M}(H, L) \in[0,+\infty]$ (see, for example, [6, 8] or [18]). Furthermore

$$
\sup _{n \in \mathbb{N}} M_{n}(H, L)=\lim _{n \rightarrow \infty} M_{n}(H, L) \quad \text { and } \quad \inf _{n \in \mathbb{N}} \bar{M}_{n}(H, L)=\lim _{n \rightarrow \infty} \bar{M}_{n}(H, L) .
$$

The limits $M(H, L), \bar{M}(H, L)$ above are called the Chebyshev constant and the dual Chebyshev constant of $L$ with respect to $H$.

If $X$ is a Hausdorff topological space, let us denote by $\mathfrak{M}(X)$ the set of positive, regular Borel measures on $X$ and by $\mathfrak{R}_{1}(X)$ the subset of probability measures. The notation $\mathfrak{M}_{1}^{\#}(X)$ is used for probability measures with finite support. Given a set $H \subseteq X$, for the subfamily of measures concentrated on $H$ (or supported on $H$, in case $H$ is closed, see [9, pp. 144-146]) we use the analogous notations $\mathfrak{M}(H), \mathfrak{M}_{1}(H)$ and $\mathfrak{M}_{1}^{\#}(H)$, respectively. The potential of a measure $\mu \in \mathfrak{M}(X)$ is

$$
U^{\mu}(x):=\int_{X} k(x, y) \mathrm{d} \mu(y) .
$$

In the classical potential theoretic literature various notions of energies appear. Already Fuglede [10] and Ohtsuka [18] introduced the following two-variate versions of energies (see also [6]).

Definition 2: Let $H, L \subset X$ be fixed, and $\mu \in \mathfrak{M}_{1}(X)$ be arbitrary. First put

$$
Q(\mu, H):=\sup _{x \in H} U^{\mu}(x), \quad \text { and also } \quad \underline{Q}(\mu, H):=\inf _{x \in H} U^{\mu}(x) .
$$

Then the quasi-uniform energy and dual quasi-uniform energy of $L$ with respect to $H$ are

$$
q(H, L):=\inf _{\mu \in \mathfrak{M}_{1}(H)} Q(\mu, L) \quad \text { and } \quad \underline{q}(H, L):=\sup _{\nu \in \mathfrak{M}_{1}(H)} \underline{Q}(\nu, L) .
$$

We use the notation $M(H):=M(H, H), \bar{M}(H):=\bar{M}(H, H), \underline{q}(H):=\underline{q}(H, H)$ and $q(H):=q(H, H)$ for the diagonal (classical) cases of the quantities given in Definitions 1 and 2.

REMARK 3. It is not surprising that in general the quantities $M(H), q(H)$ et cetera, do not posses any monotonicity properties as functions of the set $H$. The worst consequence of this is the lack of good "inner regularity" properties, for example $q(H)=\inf _{K \in H} q(K)$ 
fails to hold (we use the abbreviation $K \Subset H$ to express that $K$ is a compact subset of $H$ ). However, fixing one variable the functions $M(H, L), \bar{M}(L, H), \underline{q}(H, L)$ and $q(L, H)$ are increasing with respect to $H$ and decreasing with respect to $L$, and the above mentioned problem disappears. This particularly explains the relevance and importance of the above two-variable definitions to our subject, see also [6].

DEFINITION 4: For arbitrary subsets $H, L \subset X$ the $n^{\text {th }}$ (extended) rendezvous set of $L$ with respect to $H$ is

$$
\begin{aligned}
R_{n}(H, L) & :=\bigcap_{w_{1}, \ldots, w_{n} \in H} \overline{\operatorname{conv}}\left\{p_{n}(x):=\frac{1}{n} \sum_{j=1}^{n} k\left(x, w_{j}\right): x \in L\right\} \\
R_{n}(H) & :=R_{n}(H, H) .
\end{aligned}
$$

Correspondingly, one defines

$$
R(H, L):=\bigcap_{n=1}^{\infty} R_{n}(H, L), \quad R(H):=R(H, H) .
$$

Similarly, one defines the (extended) average set of $L$ with respect to $H$ as

$$
A(H, L):=\bigcap_{\mu \in \mathscr{N}_{1}(H)} \overline{\operatorname{conv}}\left\{U^{\mu}(x): x \in L\right\}, \quad A(H):=A(H, H) .
$$

REMARK 5. Denoting the interval

$$
A(\mu, L):=[Q(\mu, L), Q(\mu, L)]=\overline{\operatorname{conv}}\left\{U^{\mu}(x): x \in L\right\},
$$

we see that $R_{n}(H, L), R(H, L)$ and $A(H, L)$ are all of the form $\bigcap_{\mu} A(\mu, H)$, with $\mu$ ranging over all averages of $n$ Dirac measures at points of $H$, over $\mathfrak{M}_{1}^{\#}(H)$ and over all of $\mathfrak{M}_{1}(H)$, respectively.

REMARK 6. Let us explain how the above notions relate to the usual definitions of rendezvous numbers or average numbers. Suppose that $(X, k)$ is a metric space and that the set $L$ is compact. Then there is no need for the closure in the above definitions, since in this case the potential $U^{\mu}$ is continuous, so the set $A(\mu, L)$, being the continuous image of the compact set $L$, is compact. This means that a number $r \in \mathbb{R}_{+}$belongs to $R(H, L)$ if and only if for any finite system of (not necessarily distinct) points $x_{1}, \ldots, x_{n} \in H$ one finds points $y, z \in L$ satisfying

$$
\frac{1}{n} \sum_{j=1}^{n} k\left(y, x_{j}\right) \leqslant r \quad \text { and } \quad \frac{1}{n} \sum_{j=1}^{n} k\left(z, x_{j}\right) \geqslant r .
$$

This is the usual definition of weak rendezvous numbers in metric spaces (see [22]). In the next step, we can assume that $L$ is connected. In this case, (8) is further equivalent 
to the existence of a "rendezvous point" $x \in L$ with

$$
\frac{1}{n} \sum_{j=1}^{n} k\left(x, x_{j}\right)=r
$$

Of course in the above reasoning an arbitrary probability measure $\mu$ can replace the average of Dirac measures. To sum up, for compact and connected sets $L$ of metric spaces, $R(L)$ (and $A(L)$ ) is a single point, and it is the classical rendezvous (or average) number of $L$ (results of Gross [13], Elton and Stadje [21]). For further discussion and examples see [6].

From the above definitions it is easy to identify the lower and upper endpoints of the rendezvous and the average intervals (see [6]).

Proposition 7. For arbitrary subsets $H, L \subset X$ we have

$$
\begin{aligned}
R_{n}(H, L) & =\left[M_{n}(H, L), \bar{M}_{n}(H, L)\right], & R_{n}(H) & =\left[M_{n}(H), \bar{M}_{n}(H)\right], \\
R(H, L) & =[M(H, L), \bar{M}(H, L)], & R(H) & =[M(H), \bar{M}(H)], \\
A(H, L) & =[q(H, L), q(H, L)], & & A(H)=[q(H), q(H)] .
\end{aligned}
$$

The questions of existence and uniqueness of rendezvous or average numbers, are two naturally posed problems, which were investigated in [6] in the potential theoretic framework on locally compact spaces. In fact, non-emptyness of the rendezvous, respectively the average intervals means that in the above formulation (10) the formal lower endpoints of the intervals do not exceed the upper endpoints (we use the convention $[a, b]=\emptyset$ if $a>b$ ). While uniqueness is the same as that the respective interval reduces to one point. We recall the following two results from [6].

THEOREM 8. Let $X$ be a locally compact Hausdorf space, $\emptyset \neq H \subset L \subset X$ be arbitrary, and let $k$ be any nonnegative, symmetric kernel on $X$. Then the intervals $R_{n}(H, L), R(H, L)$ and $A(H, L)$ are nonempty.

THEOREM 9. Let $X$ be any locally compact Hausdorff topological space, $k$ be any lower semicontinuous, nonnegative, symmetric kernel function, and $\emptyset \neq K \Subset X$ compact. Then $A(K)$ consists of one single point. Furthermore, if $k$ is continuous, then even $R(K)$ consists of only one point.

When the rendezvous or the average interval $R(K)$ respectively $A(K)$ consists of one point only, this single point is denoted by $r(K)$ or $a(K)$, respectively.

Let us close this introduction with a few remarks to explain the idea of the present approach. Investigating the polarisation constant problem, it was found in [1] that for certain cases the Chebyshev constants of the unit spheres $S^{2}$ and $S^{3}$ appear as polarisation constants. The arising questions led to the systematic analysis of Chebyshev constants and also transfinite diameters and minimal energies in the general potential theoretical 
framework [5]. Meanwhile, the second author took part in working out a general approach which might be termed as appropriate averaging over $S^{n}$, to estimate the polarisation constant $[19,20]$. However, it turned out that part of the results achieved through such a potential theory flavoured approach, were already obtained by García-Vázquez and Villa [11], who used Gross' Theorem on the existence of rendezvous numbers successfully in the context. This suggested that perhaps there is a way to relate the two methods, or even the underlying theories, that is, potential theory and rendezvous numbers. Our paper stems from this observation.

\section{RENDEZVOUS NUMBERS FOR NORMED SPACES}

In the last decade many results were obtained regarding the numerical values of rendezvous numbers of concrete spaces and sets, see for example, $[2,11,16,23,24]$. In this context, the following terminology was introduced.

Definition 10: Let $(X,\|\cdot\|)$ be a normed space with closed unit ball $B_{X}$ and unit sphere $S_{X}$. Considering $S_{X}$ with the norm-distance, the rendezvous numbers of the arising metric space are called the rendezvous numbers of the normed space $X$. Accordingly, we use the script notation

$$
\mathcal{R}_{n}(X):=R_{n}\left(S_{X}\right), \quad \mathcal{R}(X):=R\left(S_{X}\right) \quad \text { and } \quad \mathcal{A}(X):=A\left(S_{X}\right) .
$$

It is clear that for finite dimensional normed spaces the above notion is a special case of the general notion described in Section 1. However, for infinite dimensional normed spaces the metric space $S_{X}$ will not be locally compact, as is usually assumed in the potential theoretic setup. There are two ways to tackle this, one being the extension of the theory to not necessarily locally compact but metric spaces, as is done in [7]. There we assume that the topology arises from a metric, but relax on local compactness. Conversely, in a number of cases it is possible to consider a different topology, in which the metric is still lower semicontinuous, while $S_{X}$ becomes locally compact, hence Fugledetype general potential theory applies. Note that in this case the topology is not the metric topology, which deserves some care when working with the theory. In particular, the average sets $A(H, L)$, referring to regular Borel measures of the space, may be different for different topologies. On the other hand, for a fixed kernel

$$
R(H, L)=[M(H, L), \bar{M}(H, L)]
$$

is independent of any topology.

Proposition 11. Let $X$ be any abstract set, $k \geqslant 0$ be a symmetric function from $X \times X$ to $\mathbb{R}_{+} \cup\{+\infty\}$, and $\emptyset \neq H \subset L \subset X$ be arbitrary subsets. Then $R(H, L) \neq \emptyset$.

Proof: The definition of rendezvous intervals, as well as the corresponding statement in Proposition 7, are independent of the topology of the underlying space. Therefore, we can just take the discrete topology of $X$, and note that the kernel $k$ becomes 
continuous, hence lower semicontinuous, in this topology. Thus Theorem 8 applies and $R(H, L) \neq \emptyset$.

In view of Proposition 11, we trivially obtain the following.

Corollary 12 . Let $X$ be any normed space. Then the rendezvous set $\mathcal{R}(X)$ is non-empty.

This seemingly contradicts to some assertions on nonexistence in the literature: the reason is that we considered also the closure in the definition of the rendezvous and average intervals. The above proposition shows that in the context taking the closure is also helpful. Note that Baronti, Casini and Papini [2] have already considered the closed version of the rendezvous sets, at least in normed spaces but they focused on the question of "attaining" the rendezvous numbers. In such investigations the geometry of Banach spaces plays an important role.

On the other hand, uniqueness, so nicely obtained for locally compact spaces, continuous kernels and compact sets, can not be concluded as already shown by a couple of examples in the literature (see $[14,15,16]$ ).

\section{Average NUmbers for NORMED SPACES}

For compact sets $K$ and continuous kernels it is already known that $A(K)=R(K)$, and that there are counterexamples showing that in general compactness is needed (compare [6, Section 6]). Nevertheless, the assertion remains valid in normed spaces, too.

TheOREM 13. Let $X$ be any normed space. Then we have $\mathcal{A}(X)=\mathcal{R}(X) \neq \emptyset$.

For not necessarily finite dimensional Banach spaces, we do not have the means to restrict considerations to compactly supported measures only. Instead, we prove the following result, whose easy consequence is the above theorem.

THEOREM 14. Let $(Y, d)$ be a metric space. Assume that the kernel $k$ is positive, symmetric and bounded and that $\{k(\cdot, y): y \in Y\}$ is uniformly equicontinuous on $(Y, d)$. Then we have $A(Y)=R(Y) \neq \emptyset$.

LEMMA 15. Assume that the kernel $k$ is positive, symmetric and bounded and that $\{k(\cdot, y): y \in Y\}$ is uniformly equicontinuous on $Y$. Let $\mu \in \mathfrak{M}_{1}(Y)$ and $\varepsilon>0$ be given arbitrarily. Then there exist $m \in \mathbb{N}$ and points $x_{j} \in Y(j=1, \ldots, m)$ such that the potential $U^{\nu}$ of the measure $\nu:=(1 / m) \sum_{j=1}^{m} \delta_{x_{j}}$ approximates $U^{\mu}$ within $\varepsilon$ uniformly
on $Y$.

To prove this lemma we need the following elementary result.

LEMMA 16. For any $\varepsilon>0$ and any finitely supported probability measure $\nu$, there exists a probability measure of the form $\mu=(1 / m) \sum_{i=1}^{m} \delta_{z_{i}}$ having the same support as $\nu$ and satisfying $(1-\varepsilon) \nu \leqslant \mu \leqslant(1+\varepsilon) \nu$. 
Proof: [Proof of Lemma 15] Without loss of generality we can assume that $k \leqslant 1$, and hence $U^{\sigma} \leqslant 1$ for all probability measures $\sigma$. By the assumptions we find an $r>0$, such that $\left|k\left(x^{\prime}, y\right)-k\left(x^{\prime \prime}, y\right)\right|<\varepsilon / 2$, if $d\left(x^{\prime}, x^{\prime \prime}\right)<r$. As $\mu$ is a regular Borel measure, for any given $\eta>0$ there exists $K \Subset \operatorname{supp} \mu \subset Y$ with $\mu(K) \geqslant 1-\eta$. Take now $\nu_{K}:=\mu_{K} /\left\|\mu_{K}\right\|$ (with $\mu_{K}$ being the trace of $\mu$ on $K$ ) so that $\nu_{K} \in \mathfrak{M}_{1}(K)$. Note that

$$
\left|U^{\mu}(x)-U^{\mu_{K}}(x)\right| \leqslant \sup _{y \in Y} k(x, y) \cdot\left\|\mu-\mu_{K}\right\| \leqslant \eta
$$

and

$$
\left|U^{\nu_{K}}(x)-U^{\mu_{K}}(x)\right| \leqslant\left|U^{\nu_{K}}(x)\right| \cdot\left(1-\left\|\mu_{K}\right\|\right) \leqslant \eta .
$$

Consider now the covering of the compact set $K \Subset Y$ by open balls $B(y, r)$, with $y \in K$. By compactness there exist a finite sub-covering, that is, there exist $n \in \mathbb{N}, y_{j} \in K$, $B_{j}:=B\left(y_{j}, r\right)(j=1, \ldots, n)$ satisfying $K \subset \bigcup_{j=1}^{n} B_{j}$. Put $D_{1}:=B_{1}$, and for $j=2, \ldots, n$ put

$$
D_{j}:=B_{j} \backslash \bigcup_{i=1}^{j-1} B_{i}, \alpha_{j}:=\nu_{K}\left(D_{j}\right) \geqslant 0
$$

Clearly $\sum_{j=1}^{n} \alpha_{j}=\nu_{K}(K)=1$. Consider the finitely supported measure

$$
\sigma:=\sum_{j=1}^{n} \alpha_{j} \delta_{y_{j}} \in \mathfrak{M}_{1}(K)
$$

Then we have

$$
\begin{aligned}
\left|U^{\nu_{K}}(x)-U^{\sigma}(x)\right| & =\left|\sum_{j=1}^{n} \int_{D_{j}} k(x, y)-k\left(x, y_{j}\right) \mathrm{d} \nu_{K}(y)\right| \\
& \leqslant \sum_{j=1}^{n} \alpha_{j} \sup _{y \in D_{j} \subset B_{j}}\left|k(x, y)-k\left(x, y_{j}\right)\right| \leqslant \varepsilon / 2 .
\end{aligned}
$$

Finally, the application of Lemma 16 to $\sigma$ yields an approximating measure $\nu:=(1 / m) \sum_{j=1}^{m} \delta_{x_{j}}$ with $m \in \mathbb{N}$ and points $x_{j} \in K(j=1, \ldots, m)$ so that $(1-\eta) \sigma \leqslant \nu$ $\leqslant(1+\eta) \sigma$, and thus

$$
\left|U^{\sigma}(x)-U^{\nu}(x)\right| \leqslant \eta\left|U^{\sigma}(x)\right| \leqslant \eta
$$

Collecting all the above, we find

$$
\left|U^{\mu}(x)-U^{\nu}(x)\right| \leqslant 3 \eta+\varepsilon / 2<\varepsilon
$$

if $\eta<\varepsilon / 6$.

Proof: [Proof of Theorem 14] It is obvious that $A(Y) \subset R(Y)$, hence it suffices to show the converse inclusion. Let $\mu \in \mathfrak{M}_{1}(Y)$ be arbitrary and consider $A(\mu, Y)=[a, b]$, 
(where $a=\underline{Q}(\mu, Y), b=Q(\mu, Y))$. Let us take $\varepsilon:=1 / n$ and look at the measure $\nu:=\nu_{n}$ provided by Lemma 15 to $\mu$ and $\varepsilon$. Since the potential functions are uniformly close to each other on $Y$, their infima and suprema are also within $\varepsilon$ : that is,

$$
|\underline{Q}(\mu, Y)-\underline{Q}(\nu, Y)| \leqslant \varepsilon,|Q(\mu, Y)-Q(\nu, Y)| \leqslant \varepsilon .
$$

In other words,

$$
A\left(\nu_{n}, Y\right) \subset\left[a-\frac{1}{n}, b+\frac{1}{n}\right]
$$

and thus

$$
\bigcap_{n=1}^{\infty} A\left(\nu_{n}, Y\right) \subset[a, b]=A(\mu, Y) .
$$

It follows that

$$
R(Y)=\bigcap_{\nu \in \mathfrak{M}_{1}^{\#}(Y)} A(\nu, Y) \subset A(\mu, Y)
$$

for all $\mu \in \mathfrak{M}_{1}(Y)$, hence

$$
R(Y) \subset \bigcap_{\mu \in \mathfrak{M}_{1}(Y)} A(\mu, Y)=A(Y)
$$

and the theorem is proved.

REMARK 17. The assumptions of Theorem 14 are satisfied, for instance, when $(X, d)$ is a metrisable topological vector space and $k(x, y)=f(d(x, y))$, where $f$ is a continuous function (see Section 4 below).

LEMMA 18. Let $X$ be a (not necessarily locally compact) Hausdorff topological vector space, $k$ a lower semicontinuous, nonnegative, symmetric and convex kernel function on $X \times X$, and $\mu \in \mathfrak{M}_{1}(X)$. Then the potential function $U^{\mu}$ is convex.

Proof: Take any $x, y, z \in X$ with $z=\alpha x+(1-\alpha) y$, where $0 \leqslant \alpha \leqslant 1$. We then have

$$
\begin{aligned}
U^{\mu}(z) & =\int_{X} k(z, w) \mathrm{d} \mu(w) \leqslant \int_{X}(\alpha k(x, w)+(1-\alpha) k(y, w)) \mathrm{d} \mu(w) \\
& =\alpha U^{\mu}(x)+(1-\alpha) U^{\mu}(y)
\end{aligned}
$$

and that was to be proved.

LEMma 19. Let $X$ be a (not necessarily locally compact) Hausdorff topological vector space, $k$ a lower semicontinuous, nonnegative, symmetric and convex kernel function on $X \times X, H \subset X$ a bounded set, and $\partial H$ be its boundary. Then for any $\mu \in \mathfrak{M}_{1}(X)$ the potential function $U^{\mu}$ satisfies $\sup _{H} U^{\mu}=\sup _{\partial H} U^{\mu}$. 
PROOF: We are to show $\sup _{H} U^{\mu} \leqslant \sup _{\partial H} U^{\mu}$, the other direction being obvious. Let now $x \in H$ be arbitrary: we show that $U^{\mu}(x) \leqslant \sup _{\partial H} U^{\mu}$. Draw any straight line $\ell$ through $x$. Since $H$ is bounded, both closed half-lines of $\ell$, starting from $x$, contain boundary points of $H$; that is, if these points are $y, z \in \ell$, then $x \in[y, z]$ with $y, z$ $\in \partial H$. According to Lemma 18 , the potential is convex, which immediately yields $U^{\mu}(x)$ $\leqslant \max \left(U^{\mu}(y), U^{\mu}(z)\right) \leqslant \sup _{\partial H} U^{\mu}$.

Corollary 20. If $X$ is a normed space, then $q\left(S_{X}, S_{X}\right)=q\left(S_{X}, B_{X}\right)$ and $\bar{M}\left(S_{X}, S_{X}\right)=\bar{M}\left(S_{X}, B_{X}\right)$.

REMARK 21. Note that for the other endpoints of the average intervals generally we may have strict inequality: $\underline{q}\left(S_{X}, B_{X}\right)<\underline{q}\left(S_{X}, S_{X}\right)$. As will be seen in Theorem 23, for any $1<p<+\infty \mathcal{R}\left(\ell_{p}\right)=2^{1 / p}>1$. However, for any measure $\mu \in \mathfrak{M}_{1}\left(S_{X}\right)$, it is clear that $0 \in B_{X}$ satisfies $U^{\mu}(0)=\int_{S_{X}} 1 \mathrm{~d} \mu=1$, hence $\underline{q}\left(S_{X}, B_{X}\right) \leqslant 1$. In fact, $\underline{q}\left(S_{X}, B_{X}\right)=1$ is true, since for any two points $x, y \in B_{X}$, the corresponding measure $\nu:=\left(\delta_{x}+\delta_{y}\right) / 2$ always provides, by the triangle inequality, $\underline{Q}\left(\nu, B_{X}\right) \geqslant\|x-y\| / 2$, which can be as large as half the diameter.

REMARK 22. It is straightforward to show that

$$
[M(H), \bar{M}(H)]=R(H) \subset[\operatorname{diam}(H) / 2, \operatorname{diam}(H)]
$$

(see, for example, [13]). Indeed, $\bar{M}(H) \leqslant \operatorname{diam}(H)$ is trivial, while the lower estimate $\operatorname{diam}(H) / 2 \leqslant M(H)$ is essentially contained in the previous remark.

\section{RENDEZVOUS NUMBERS FOR $L_{p}$ SPACES}

We already know that the rendezvous interval of a Banach space is not empty. Here we identify the rendezvous, hence the average intervals of the $L_{p}$ spaces.

Let $(\Omega, \mathcal{M}, \mu)$ be a measure space. To complement the whole scale $1 \leqslant p<+\infty$, we consider $L_{p}:=L_{p}(\Omega, \mathcal{M}, \mu)$ when $0<p<1$ as well. In this case $L_{p}$ will not be a Banach space, but if endowed with the metric

$$
d(f, g)=\int_{\Omega}|f-g|^{p} \mathrm{~d} \mu,
$$

it is a complete, metrisable, topological vector space (of course we have to identify functions coinciding on $\mu$-null sets). First we calculate the rendezvous number with respect to the symmetric function $\|f-g\|:=d(f, g)^{1 / p}$ instead of the metric $d$, this fits well together with the case $p \geqslant 1$. Of course, now $S_{L_{p}}$ denotes the "unit sphere" with respect to $\|\cdot\|$ for all $0<p<+\infty$.

THEOREM 23. Let $0<p<+\infty$ be arbitrary and consider $L_{p}(\Omega, \mathcal{M}, \mu)$ over either $\mathbf{R}$ or $\mathbb{C}$. If $L_{p}$ is infinite dimensional, we have $a\left(S_{L_{p}}\right)=r\left(S_{L_{p}}\right)=2^{1 / p}$. 
Proof: The following applies for both the complex- and real-valued cases, hence we do not mention the underlying number field any more. Further we write briefly $L_{p}$ instead of $L_{p}(\Omega, \mathcal{M}, \mu)$. We already know that $R\left(S_{L_{p}}\right)$ is nonempty (see Proposition 11 ) and is a compact interval (indeed, in case $p \geqslant 1$ it is a subset of the interval $[1,2]$, see Remark 22). Moreover, we have $R\left(S_{L_{p}}\right)=\left[M\left(S_{L_{p}}\right), \bar{M}\left(S_{L_{p}}\right)\right]$, and by Theorem 13, Theorem 14 and Remark 17 we have $A\left(S_{L_{p}}\right)=R\left(S_{L_{p}}\right)$. Therefore we need only to show that $M\left(S_{L_{\mathrm{p}}}\right) \geqslant 2^{1 / p}$ and that $\bar{M}\left(S_{L_{\mathrm{p}}}\right) \leqslant 2^{1 / p}$.

Since $L_{p}$ is infinite dimensional, there exist $w_{j} \in S_{L_{p}}, j \in \mathbb{N}$ such that the sets $A_{j}:=\left\{x: x \in \Omega, w_{j}(x) \neq 0\right\}$ are pairwise disjoint. For any function $g \in L_{p}$ let us introduce the notation $\|g\|_{j}:=\left\|\chi_{A_{j}} g\right\|$.

PART 1. $M\left(S_{L_{p}}\right) \geqslant 2^{1 / p}$. With the functions $w_{j}$ and for any $f \in S_{L_{p}}$ we have

$$
\begin{aligned}
\sum_{j=1}^{n}\left\|f-w_{j}\right\| & =\sum_{j=1}^{n}\left(\sum_{k=1}^{\infty}\left\|f-w_{j}\right\|_{k}^{p}\right)^{1 / p}=\sum_{j=1}^{n}\left(\left\|f-w_{j}\right\|_{j}^{p}+\sum_{k=1, k \neq j}^{\infty}\|f\|_{k}^{p}\right)^{1 / p} \\
& =\sum_{j=1}^{n}\left(\left\|w_{j}-f\right\|_{j}^{p}+1-\|f\|_{j}^{p}\right)^{1 / p} .
\end{aligned}
$$

Now we distinguish between the cases $p<1$ and $p \geqslant 1$. First let $p<1$, then using $\|f\|_{j} \leqslant 1$ and $f \in S_{L_{p}}$, we can continue (15)

$$
\begin{aligned}
\sum_{j=1}^{n}\left\|f-w_{j}\right\| & =\sum_{j=1}^{n}\left(1-\|f\|_{j}^{p}+\left\|w_{j}-f\right\|_{j}^{p}\right)^{1 / p} \\
& \geqslant \sum_{j=1}^{n}\left(1-\|f\|_{j}^{p}+\|\| w_{j}\left\|_{j}^{p}-\right\| f \|_{j}^{p} \mid\right)^{1 / p}=\sum_{j=1}^{n}\left(1-\|f\|_{j}^{p}+\left(1-\|f\|_{j}^{p}\right)\right)^{1 / p} \\
& =2^{1 / p} \sum_{j=1}^{n}\left(1-\|f\|_{j}^{p}\right)^{1 / p} \geqslant 2^{1 / p} n \frac{(n-1)^{1 / p}}{n^{1 / p}}
\end{aligned}
$$

using again $f \in S_{L_{p}}$ and the convexity of the function $x \mapsto x^{1 / p}$ in the last step. Second, let $p \geqslant 1$, then we can write

$$
\begin{aligned}
\sum_{j=1}^{n}\left\|f-w_{j}\right\| & =\sum_{j=1}^{n}\left(1-\|f\|_{j}^{p}+\left\|w_{j}-f\right\|_{j}^{p}\right)^{1 / p} \\
& \geqslant \sum_{j=1}^{n}\left(1-\|f\|_{j}^{p}+\left(1-\|f\|_{j}\right)^{p}\right)^{1 / p} \\
& =\sum_{j=1}^{n}\left(1-\|f\|_{j}^{p}-\left(1-\|f\|_{j}\right)^{p}+2\left(1-\|f\|_{j}^{p}\right)^{p}\right)^{1 / p} \\
& \geqslant \sum_{j=1}^{n}\left(2\left(1-\|f\|_{j}\right)^{p}\right)^{1 / p}=2^{1 / p} \sum_{j=1}^{n}\left(1-\|f\|_{j}\right) \geqslant 2^{1 / p}\left(n-n^{1 / q}\right),
\end{aligned}
$$


where $1 / p+1 / q$ and using again $f \in S_{L_{\mathrm{p}}}$ and Hölder's inequality in the last step. We see that in both cases for the given $n$-point distribution (concentrated on the $w_{j} s$, $j=1, \ldots, n)$ the corresponding potential is minorised by the right hand sides divided by $n$, hence we find $M_{n}\left(S_{L_{p}}\right) \geqslant 2^{1 / p}+o_{p}(1)$ (as $\left.n \rightarrow \infty\right)$ and $M\left(S_{L_{p}}\right) \geqslant 2^{1 / p}$ follows. (Note that, for example, [11] calculates even the exact value of $\mathcal{R}\left(\ell_{1}^{n}(\mathbb{C})\right)$, but here the task is a little bit different.)

PART 2. $\bar{M}\left(S_{L_{p}}\right) \leqslant 2^{1 / p}$. Let $n \in \mathbb{N}$ and consider the same functions $w_{j}, j \in \mathbb{N}$ as in the first part. Then for any point $f \in S_{L_{p}}$ and for any given parameter $\eta>0$ we have, by (15), for $p \geqslant 1$

$$
\begin{aligned}
\sum_{j=1}^{n}\left\|f-w_{j}\right\| & \leqslant \sum_{j=1}^{n}\left(1+\left(1+\|f\|_{j}\right)^{p}\right)^{1 / p} \\
& \leqslant \sum_{j:\|f\|_{j}>\eta}\left(1+2^{p}\right)^{1 / p}+\sum_{j:\|f\|_{j} \leqslant \eta}\left(1+(1+\eta)^{p}\right)^{1 / p} \\
& \leqslant \frac{1}{\eta^{p}}\left(1+2^{p}\right)^{1 / p}+n 2^{1 / p}(1+\eta)
\end{aligned}
$$

and for $p<1$

$$
\begin{aligned}
\sum_{j=1}^{n}\left\|f-w_{j}\right\| & \leqslant \sum_{j=1}^{n}\left(1+\left(1+\|f\|_{j}^{p}\right)\right)^{1 / p} \\
& \leqslant \sum_{j:\|f\|_{j}>\eta} 3^{1 / p}+\sum_{j:\|f\|_{j} \leqslant \eta}\left(1+\left(1+\eta^{p}\right)\right)^{1 / p} \leqslant \frac{3^{1 / p}}{\eta^{p}}+n 2^{1 / p}\left(1+\eta^{p}\right)^{1 / p} .
\end{aligned}
$$

Choosing, for example, $\eta:=n^{-1 / 2 p}$, we obtain the estimate $U^{\nu}(f) \leqslant 2^{1 / p}+o_{p}(1)$ (as $\left.n \rightarrow+\infty, \forall f \in S_{L_{p}}\right)$ for the measure $\nu:=(1 / n) \sum_{j=1}^{n} \delta_{w_{j}}$. It follows that for the given $n$-point distribution $\nu$ we have

$$
\bar{M}_{n}\left(S_{L_{p}}\right) \leqslant Q\left(\nu, S_{L_{p}}\right)=2^{1 / p}+o_{p}(1) \rightarrow 2^{1 / p}(n \rightarrow \infty),
$$

and so even $\bar{M}\left(S_{L_{p}}\right) \leqslant 2^{1 / p}$.

Note that for $p \geqslant 1$ already Lin [16] showed that $\mathcal{R}\left(\ell_{p}\right) \subseteq\left\{2^{1 / p}\right\}$ for "strict" rendezvous numbers (actually by a similar argument). So this and the non-emptyness of the rendezvous interval (Corollary 12) give the above result for $\ell_{p}(1 \leqslant p<+\infty)$.

Corollary 24. (Wolf, Lin) Let $\mathcal{H}$ be an infinite dimensional Hilbert space over any of the number fields $\mathbb{R}$ or $\mathbb{C}$. Then we have $\mathcal{A}(\mathcal{H})=\mathcal{R}(\mathcal{H})=\{\sqrt{2}\}$.

REMARK 25. In the above proof we actually used the same point distribution in both parts of the proof, therefore we have proved the existence of $\varepsilon$-quasi-invariant measures. We say that there exist $\varepsilon$-quasi-invariant measures see [7, Definition 5.9] for the kernel $k$ on $S$, if for all $\varepsilon>0$ there is $\nu \in \mathfrak{M}_{1}(S)$ satisfying $Q(\nu, S)-\underline{Q}(\nu, S) \leqslant \varepsilon$. Generally, if 
$S$ is compact, by weak*-compactness we obtain the existence of a true invariant measure $\mu \in \mathfrak{M}_{1}(S)$, that is, whose potential $U^{\mu}$ is constant on $S$. Of course, for $S=S_{\ell_{p}}$ this is not the case. But as we saw above there exist $\varepsilon$-quasi-invariant measures, and by [7, Proposition 5.11] it is already enough to conclude that the average interval reduces to one point.

THEOREM 26. Let $0<p<1$ be arbitrary and $L_{p}(\Omega, \mathcal{M}, \mu)$ be the vector space of p-integrable functions endowed with the metric $d$ defined in (14). For the rendezvous interval of the unit ball $S_{L_{p}}$ of $L_{p}$ we have $A\left(S_{L_{p}}\right)=R\left(S_{L_{p}}\right)=\{2\}$.

Proof: By Remark 22 we know $\bar{M}\left(S_{L_{p}}\right) \leqslant 2$. So we only need to estimate the lower endpoint of $R\left(S_{L_{p}}\right)$ from below. This can be done analogously to (15) and (16) by considering the functions $w_{j}$ and sets $A_{j}$ used in the proof of Theorem 23. Let us further use the abbreviation $\|f\|_{j}:=\int_{A_{j}}|f|^{p} \mathrm{~d} \mu$. For an arbitrary $f \in S_{L_{p}}$ we can write

$$
\begin{aligned}
\sum_{j=1}^{n} d\left(f, w_{j}\right) & =\sum_{j=1}^{n}\left(\sum_{k=1}^{\infty}\left\|f-w_{j}\right\|_{k}\right)=\sum_{j=1}^{n}\left(\|\| f-w_{j}\left\|_{j}+\sum_{k=1, k \neq j}^{\infty}\right\| f \|_{k}\right) \\
& =\sum_{j=1}^{n}\left(1-\|f\|_{j}+\left\|w_{j}-f\right\|_{j}\right) \\
& \geqslant \sum_{j=1}^{n}\left(1-\|f\|_{j}+1-\|f\|_{j}\right)=2 \sum_{j=1}^{n}\left(1-\|f\|_{j}\right) \geqslant 2(n-1) .
\end{aligned}
$$

Therefore $M_{n}\left(S_{L_{p}}\right) \geqslant 2-1 / n$, hence $M\left(S_{L_{p}}\right) \geqslant 2$.

It was already pointed out by $\operatorname{Lin}$ in [16] that $\lim _{n \rightarrow \infty} r\left(S_{\ell_{p}^{n}}\right)=2^{1 / p}$, if $1 \leqslant p<+\infty$. The following result, inspired by an analogous argument of Garcia-Vázquez and Villa [11], explains this phenomenon in view of Theorem 23 above.

THEOREM 27. Let $X$ be a normed space and $X_{n}$ an increasing sequence of subspaces such that $\bigcup_{n=1}^{\infty} X_{n}$ is dense in $X$. Suppose that $\left(X_{n},\|\cdot\|_{n}\right)$ is a normed space and $\rho_{n} \in R\left(S_{\left(X_{n},\|\cdot\|_{n}\right)}\right)$. Assume that

$$
\lim _{n \rightarrow \infty} \sup _{x \in X_{n} \cap S_{X}}\left|1-\|x\|_{n}\right|=0 .
$$

Then any accumulation point $\rho$ of the sequence $\rho_{n}$ belongs to $R\left(S_{X}\right)$.

ProOF: Let $\rho$ be an accumulation point of the sequence $\rho_{n}$. Assume without loss of generality that $\rho_{n} \rightarrow \rho$. Let $\varepsilon>0$ be given. Then for sufficiently large $n \geqslant n_{0}(\varepsilon)$ we have $\left[\rho_{n}-\varepsilon, \rho_{n}+\varepsilon\right] \subset[\rho-2 \varepsilon, \rho+2 \varepsilon]$.

By definition $\rho_{n} \in \mathcal{R}_{m}\left(X_{n}\right)$ for all $m \in \mathbb{N}$. Let $m \in \mathbb{N}$ be fixed, and $x_{1}, \ldots, x_{m} \in S_{X}$ be arbitrary. Take any $y_{j} \in X_{n} \cap S_{X}$ with $\left\|y_{j}-x_{j}\right\| \leqslant \varepsilon$. Such $y_{j}$ exists in view of the denseness of $\bigcup_{n=1}^{\infty} X_{n}$ in $X$. By assumption, we have $\left|\|z\|-\|z\|_{n}\right| \leqslant \varepsilon\|z\|$ for all sufficiently 
large $n \geqslant n_{1} \geqslant n_{0}$ and all $z \in X_{n}$. In particular, $\left|1-\left\|y_{j}\right\|_{n}\right| \leqslant \varepsilon$. By definition of the rendezvous interval $\mathcal{R}_{m}\left(X_{n}\right)$, we find $z_{n} \in S_{\left(X_{n},\|\cdot\|_{n}\right)}$ satisfying

$$
\frac{1}{m} \sum_{j=1}^{m}\left\|\frac{y_{j}}{\left\|y_{j}\right\|_{n}}-z_{n}\right\|_{n} \in\left[\rho_{n}-\varepsilon, \rho_{n}+\varepsilon\right] \subset[\rho-2 \varepsilon, \rho+2 \varepsilon] \text {. }
$$

According to the above, for all $n \geqslant n_{1}$ we have

$$
\left\|z_{n}-\frac{z_{n}}{\left\|z_{n}\right\|}\right\|_{n}=\left|1-\frac{1}{\left\|z_{n}\right\|}\right| \cdot\left\|z_{n}\right\|_{n}=\left|1-\frac{\left\|z_{n}\right\|_{n}}{\left\|z_{n}\right\|}\right| \leqslant \varepsilon,
$$

and

$$
\left\|y_{j}-\frac{y_{j}}{\left\|y_{j}\right\|_{n}}\right\|_{n}=\left|1-\frac{1}{\left\|y_{j}\right\|_{n}}\right| \cdot\left\|y_{j}\right\|_{n}=\left|\left\|y_{j}\right\|_{n}-1\right| \leqslant \varepsilon
$$

Using these two inequalities in (19) we obtain

$$
\frac{1}{m} \sum_{j=1}^{m}\left\|y_{j}-\frac{z_{n}}{\left\|z_{n}\right\|}\right\|_{n} \in[\rho-4 \varepsilon, \rho+4 \varepsilon] \text {. }
$$

For $n>n_{1}$ we also know

$$
\left|\left\|y_{j}-\frac{z_{n}}{\left\|z_{n}\right\|}\right\|-\left\|y_{j}-\frac{z_{n}}{\left\|z_{n}\right\|}\right\|_{n}\right| \leqslant \varepsilon \cdot\left\|y_{j}-\frac{z_{n}}{\left\|z_{n}\right\|}\right\| \leqslant 2 \varepsilon,
$$

therefore we can write

$$
\frac{1}{m} \sum_{j=1}^{m}\left\|y_{j}-\frac{z_{n}}{\left\|z_{n}\right\|}\right\| \in[\rho-6 \varepsilon, \rho+6 \varepsilon] \text { and } \frac{1}{m} \sum_{j=1}^{m}\left\|x_{j}-\frac{z_{n}}{\left\|z_{n}\right\|}\right\| \in[\rho-7 \varepsilon, \rho+7 \varepsilon] .
$$

This shows $\rho \in R_{m}\left(S_{X}\right)$, which being valid for all $m$, gives $\rho \in R\left(S_{X}\right)$.

This theorem immediately gives the following corollary.

Corollary 28. Let $X$ be a normed space and $X_{n}$ an increasing sequence of finite dimensional subspaces such that $\bigcup_{n=1}^{\infty} X_{n}$ is dense in $X$. Let $\rho$ be an accumulation point of the sequence $r\left(S_{X_{n}}\right)\left(r\left(S_{X_{n}}\right)\right.$ exists uniquely by the compactness of $\left.S_{X_{n}}\right)$. Then $\rho \in \mathcal{R}(X)$.

\section{Chebyshev Centres, entropy and Rendezvous numbers}

DEFINITION 29: Let $K \subset X$ be a compact, convex subset of some normed space $X$, with $d$ being the metric induced by the norm. Then the Chebyshev centre $c:=c(K) \in K$ and the Chebyshev out-radius $\rho:=\rho(K)$ of the set $K$ are the centre and the radius, respectively, of the closed ball $\bar{B}:=\bar{B}(c, \rho)$ of minimal radius with $c \in K \subset \bar{B}(c, \rho)$. 
Clearly, for any compact $K$ such a minimal ball always exists, and for convex sets it is even unique. Note that it is important in the definition that $c$ be chosen within $K$; for discussion see $[3,4,12]$.

Quoting private communication from Esther and George Szekeres, in [4] Cleary, Morris and Yost present the following beautiful result with a nice, direct elementary proof. Here we present our even shorter version relying on the potential theoretical background developed.

ThEOREM 30. (E. and G. Szekeres) Let $K \subset X$ be a compact, convex subset of some normed space $X$, with $d$ being the metric induced by the norm. Then the Chebyshev radius and the rendezvous number of the set $K$ equal: $r(K)=\rho(K)$.

Proof: Existence and uniqueness of $R(K)=\{r(K)\}$ and also the equality $R(K)=A(K)$ are already known from Theorems 8 and 9. Further, if $c$ is a Chebyshev centre of $K$, then to all points $x \in K$ we have $\|x-c\| \leqslant \rho$. Hence for any probability measure $\mu \in \mathfrak{M}_{1}(K)$ also the potential satisfies $U^{\mu}(c) \leqslant \rho(K)$. It follows that $\underline{Q}(\mu, K) \leqslant \rho$ for all $\mu \in \mathfrak{M}_{1}(K)$, hence also $r(K)=\underline{q}(K) \leqslant \rho(K)$.

Conversely, for $\varepsilon>0$ let $y_{j} \in K$ be arbitrary points $(j=1, \ldots, n)$ satisfying $Q(\nu, K)<\bar{M}(K)+\varepsilon$ with $\nu:=(1 / n) \sum_{j=1}^{n} \delta_{y_{j}}$. As $K$ is convex, it contains the convex combination $y:=(1 / n) \sum_{j=1}^{n} y_{j} \in K$ of the given points. Thus by the convexity of the norm for arbitrary $x \in K$ the estimate

$$
\|y-x\| \leqslant \frac{1}{n} \sum_{j=1}^{n}\left\|y_{j}-x\right\|=U^{\nu}(x) \leqslant \bar{M}(K)+\varepsilon
$$

holds. Hence $\bar{B}(y, \bar{M}(K)+\varepsilon)$ covers $K$ and $\rho(K) \leqslant \bar{M}(K)+\varepsilon$, which implies also

$$
\rho(K) \leqslant \bar{M}(K)=q(K)=r(K) .
$$

Recall that for a positive number $t>0$ and a set $H \subset X$ of a metric space $X$ with metric $d$ the $t$-covering number $N(t, H)$ is defined as

$$
N(t, H):=\min \left\{n \in \mathbb{N}: \exists y_{j} \in H(j=1, \ldots, n) \text { such that } H \subset \bigcup_{j=1}^{n} B\left(y_{j}, t\right)\right\} .
$$

If there is no finite set of balls of radius $t$ which can cover the set $H$, then we say that $N(t, H)=+\infty$. Similarly, if $H, L \subset X$, then

$$
N(t, H, L):=\min \left\{n \in \mathbb{N}: \exists y_{j} \in H(j=1, \ldots, n) \text { such that } L \subset \bigcup_{j=1}^{n} B\left(y_{j}, t\right)\right\}
$$

with $\min \emptyset=+\infty$ being in effect again. The next observation is almost obvious. 
Proposition 31. Let $t>0$ and $H, L \subset X$. We have $\bar{M}_{n}(H, L) \geqslant t$ for all $n<N(t, H, L)$. In particular, if $N(t, H, L)=+\infty$, then $t \leqslant \bar{M}(H, L)=\sup R(H, L)$.

Proof: Since $n<N(t, H, L)$, for any system of points $y_{j} \in H(j=1, \ldots, n)$, there exists some point $x \in L$ so that $d\left(x, y_{j}\right) \geqslant t$ for all $j=1, \ldots, n$. Therefore, $\sup _{x \in L} \sum_{j=1}^{n} d\left(x, y_{j}\right) \geqslant n t$ holds for all systems of $n$ points, hence $\bar{M}_{n}(H, L) \geqslant t$, and (10) concludes the proof.

Recall that a set $H \subset X$ is called totally bounded, if $N(t, H)<+\infty$ for all $t>0$. In Banach spaces this is the same as the conditional compactness of $H$, that is, that $\bar{H} \Subset X$ is compact set. The proposition shows that for subsets $H$ which are not totally bounded, there is always a positive lower bound of $\bar{M}(H, H)$. The above froposition, however easy, provides an essential help in describing some rendezvous numbers. For instance, there is an elegant interpretation of the following result.

THEOREM 32. Let $K$ be a compact Hausdorff topological space without isolated points, and consider $C(K)$ the Banach space of real-or complex-valued continuous functions over $K$. Then we have $\bar{M}\left(S_{C(K)}\right)=2$.

Proof: Denote by $S$ the unit sphere of $C(K)$. We show that $N(t, S, S)=+\infty$ for $0<t<2$, then by Proposition $31 \bar{M}(S) \geqslant t$ hence $\bar{M}(S) \geqslant 2$ will follow. Then, by Remark 22, we must actually have $\bar{M}(S)=2$.

So let $0<t<2$ and $f_{1}, \ldots f_{m} \in S$. Further let $x_{j} \in K$ be one of the maximum points of $\left|f_{j}\right|$, that is, $\left|f_{j}\left(x_{j}\right)\right|=1$ and take $\varepsilon>0$ small such that $t+\varepsilon<2$. By continuity, there exist neighbourhoods $G_{j}$ of $x_{j}$ with $\left|f_{j}\left(x_{j}\right)-f_{j}(y)\right|<\varepsilon,\left(\forall y \in G_{j}\right)$, for all $j=1, \ldots, m$. Take $y_{j} \in G_{j}$ distinct points ( $x_{j}$ is not an isolated point!). By Tietze's Theorem there exists a continuous function $g \in S$, such that $g\left(y_{j}\right)=-f_{j}\left(x_{j}\right)$, for all $j=1, \ldots, m$. But then $S$ can not be covered by the balls $B\left(f_{j}, t\right)$, because this particular $g$ is not covered. Thus we conclude $N(t, S, S)=+\infty$.

The above result is already present in García-Vázquez, Villa [11] and Lin [16], where the authors determine the rendezvous interval of $C(K)$. Their proofs follow the same line, we included it for the sake of illustration of the role of entropy.

The real-valued case in the following theorem is due to Wolf [24], see also Lin [16].

THEOREM 33. Let $c_{0}$ denote the Banach space of real or complex valued nullsequences. Then we have

$$
\mathcal{A}\left(c_{0}\right)=\mathcal{R}\left(c_{0}\right)=[1, \sigma]
$$

where $\sigma=3 / 2$ or $\sigma=1 / 3+2(\sqrt{3}) / \pi$ in the case of $\mathbf{R}$ respectively $\mathbb{C}$-valued sequences.

Proof: In the real case Wolf showed that $r\left(S_{\left(_{\infty}\right.}\right)=\sigma([24$, Proposition 1]), while the corresponding equality in the complex case is due to García-Vázquez and Villa. So by Corollary 28 we see that $\sigma \in \mathcal{R}\left(c_{0}\right)$. Applying the same idea as in [11, Theorem 5], 
we can split the space as $c_{0}=\mathbb{K} \times c_{0}$, where $\mathbb{K}$ is either the complex or the real scalar field. Consider the measure $\mu=\lambda \otimes \delta_{0}$, where $\lambda$ is the normalised Haar measure on $S_{\mathbb{K}}$ and $\delta_{0}$ is the Dirac measure on $c_{0}$ at the constant 0 sequence. Clearly $\mu$ is supported in $S_{c_{0}}$. In case of real-valued sequences, Wolf essentially showed $Q\left(\mu, S_{c_{0}}\right) \leqslant \sigma$ (see proof of Proposition 1 in [24]). Moreover, one can repeat the arguments from [11] to see that $Q\left(\mu, S_{c_{0}}\right) \leqslant \sigma$ in the complex case, too. So in both cases we have $q\left(S_{c_{0}}\right) \leqslant \sigma=\sigma(\mathbb{K})$ and as by Theorem 13 we know $\bar{M}\left(S_{c_{0}}\right)=q\left(S_{c_{0}}\right)$, we find $\bar{M}\left(S_{c_{0}}\right) \leqslant \sigma$. Because $\sigma \in \mathcal{R}\left(c_{0}\right)$, the only possibility is $\bar{M}\left(S_{c_{0}}\right)=\sigma$.

To calculate the lower endpoint of the rendezvous interval, let now $m \in \mathbb{N}$ be fixed and $x_{1}, \ldots, x_{m} \in S_{c_{0}}$ be arbitrary. For $\varepsilon>0$ take $n_{0} \in \mathbb{N}$ be so large that $\left|x_{j}(n)\right|<\varepsilon$ whenever $n \geqslant n_{0}$ for all $j=1, \ldots, m$. Now let $z$ be the element of $c_{0}$ being almost completely 0 but 1 at the $n_{0}$ th coordinate. Then

$$
\frac{1}{m} \sum_{j=1}^{m}\left\|x_{j}-z\right\| \leqslant 1+\varepsilon,
$$

so $M_{m}\left(S_{c_{0}}\right) \leqslant 1$, and therefore $M\left(S_{c_{0}}\right) \leqslant 1$. But then by Remark 22 we have $M\left(S_{c_{0}}\right)=1$. We have calculated the lower and upper endpoints of the rendezvous (and the average) intervals to arrive at the assertion.

\section{REFERENCES}

[1] V. Anagnostopoulos and Sz. Gy. Révész, 'Polarization constants for products of linear functionals over $\mathbb{R}^{2}$ and $\mathbb{C}^{2}$ and Chebyshev constants of the unit sphere', Publ. Math. Debrecen (to appear).

[2] M. Baronti, E. Casini and P.L. Papini, 'On average distances and the geometry of Banach spaces', Nonlinear Anal. 42 (2000), 533-541.

[3] J. Borwein and L. Kneer, 'The Hausdorff metric and Chebyshev centres,', J. Approx. Theory 28 (1980), 366-376.

[4] J.M. Cleary, S.A. Morris and D. Yost, 'Numerical geometry - numbers for shapes', Amer. Math. Monthly 93 (1986), 260-275.

[5] B. Farkas and B. Nagy, 'Transfinite diameter, Chebyshev constant, and capacity on locally compact spaces', Alfréd Rényi Institute preprint series, Hung. Acad. Sci. 7 (2004), 10.

[6] B. Farkas and Sz.Gy. Révész, 'How magical rendezvous numbers are explained by potential theory?', Alfréd Rényi Institute preprint series, Hung. Acad. Sci. 2 (2005), 21.

[7] B. Farkas and Sz.Gy. Révész, 'Rendezvous numbers of metric spaces - a potential theoretic approach', Arch. Math. (Basel) (to appear).

[8] M. Fekete, 'Über die Verteilung der Wurzeln bei gewissen algebraischen Gleichungen mit ganzahligen Koeffizienten', Math. Z. 17 (1923), 228-249.

[9] B. Fuglede, 'On the theory of potentials in locally compact spaces', Acta Math. 103 (1960), 139-215.

[10] B. Fuglede, 'Le théorème du minimax et la théorie fine du potentiel', Ann Inst. Fourier (Grenoble) 15 (1965), 65-87. 
[11] J.C. Garcia-Vázquez and R. Villa, 'The average distance property of the spaces $\ell_{\infty}^{n}(\mathbb{C})$ and $\ell_{1}^{n}(\mathbb{C})$ ', Arch. Math. 76 (2001), 222-230.

[12] A.L. Garkavi, 'On the Chebyshev centre and convex hull of a set', Uspekhi Mat. Nauk 19 (1964), 139-145.

[13] O. Gross, 'The rendezvous value of a metric space', in Advances in Game Theory, Ann. of Math. Studies 52 (Princeton University Press, N.J., 1964), pp. 49-53.

[14] A. Hinrichs, 'The average distance property of classical Banach spaces', Bull. Aust. Math. Soc. 62 (2000), 119-134.

[15] A. Hinrichs and J. Wenzel, 'The average distance property of classical Banach spaces II', Bull. Aust. Math. Soc. 65 (2002), 511-520.

[16] P.K. Lin, 'The average distance property of Banach spaces', Arch. Math. 68 (1997), 496-502.

[17] M. Ohtsuka, 'On potentials in locally compact spaces', J. Sci. Hiroshima Univ. Ser. A-I Math. 25 (1961), 135-352.

[18] M. Ohtsuka, 'On various definitions of capacity and related notions', Nagoya Math. J. 30 (1967), 121-127.

[19] A. Pappas and Sz. Gy. Révész, 'Linear polarization constants of Hilbert spaces', J. Math. Anal. Appl. 300 (2004), 129-146.

[20] Sz. Gy. Révész and Y. Sarantopoulos, 'Plank problems, polarization, and Chebyshev constants', J. Korean Math. Soc. 41 (2004), 157-174.

[21] W. Stadje, 'A property of compact, connected spaces', Arch. Math. 36 (1981), 275-280.

[22] C. Thomassen, 'The rendezvous number of a symmetric matrix and a compact connected metric space', Amer. Math. Monthly 107 (2000), 163-166.

[23] R. Wolf, 'On the average distance property in finite dimensional real Banach spaces', Bull. Austral. Math. Soc. 51 (1994), 87-101.

[24] R. Wolf, 'On the average distance property of spheres in Banach spaces', Arch. Math. 62 (1994), 338-344.

Technische Universität Darmstadt

Fachbereich Mathematik AG4

Schloßgartenstraße 7

D-64289 Darmstadt

Germany

e-mail: farkas@mathematik.tu-darmstadt.de

\author{
Alfréd Rényi Institute \\ Hungarian Academy of Sciences \\ Reáltanoda u. 13-15 \\ H-1053, Budapest \\ Hungary \\ e-mail: revesz@renyi.hu
}

